\title{
Nanoscale imaging of primary cilia with scanning ion conductance microscopy
}

\author{
Yuanshu Zhou ${ }^{1}$, Masaki Saito², Takafumi Miyamoto ${ }^{1}$, Pavel Novak ${ }^{3}$, Andrew I Shevchuk ${ }^{4}$, Yuri E \\ Korchev $^{4}$, Takeshi Fukuma ${ }^{1,5}$, Yasufumi Takahashi ${ }^{1,6^{*}}$ \\ ${ }^{1}$ Division of Electrical Engineering and Computer Science, Kanazawa University, 920-1192, Japan \\ ${ }^{2}$ Department of Molecular Pharmacology, Tohoku University Graduate School of Medicine, 2-1 Seiryo-machi, Aoba- \\ ku, Sendai 980-8575, Japan \\ ${ }^{3}$ School of Engineering and Materials Science, Queen Mary, University of London, Mile End Road, London E1 4NS, \\ United Kingdom \\ ${ }^{4}$ Department of Medicine, Imperial College London, London W12 0NN, United Kingdom \\ ${ }^{5}$ WPI Nano Life Science Institute (WPI-NanoLSI), Kanazawa University, 920-1192, Japan \\ ${ }^{6}$ Precursory Research for Embryonic Science and Technology (PRESTO), Japan Science and Technology Agency (JST), \\ Saitama 332-0012, Japan \\ * Tel: +81 76234 4866; e-mail: yasufumi@se.kanazawa-u.ac.jp
}

\begin{abstract}
Primary cilia are hair-like sensory organelles whose dimensions and location vary with cell type and culture condition. Herein, we employed scanning ion conductance microscopy (SICM) to visualize the topography of primary cilia from two different cell types. By combining SICM with fluorescence imaging, we successfully distinguished between surface cilia that project outward from the cell surface and subsurface cilia that are trapped below it. The nanoscale structure of the ciliary pocket, which cannot be easily identified using a confocal fluorescence microscope, was observed in SICM images. Furthermore, we developed a topographic reconstruction method using current-distance profiles to evaluate the relationship between set point and topography image and found that a low set point is important for detecting the true topography of a primary cilium using hopping mode SICM.
\end{abstract}

\section{Introduction}

Primary cilia are nonmotile organelles consisting of a centriole-derived basal body and a microtubulebased axoneme ${ }^{1}$. It is widely considered that the primary cilium acts as a cellular antenna that senses 
extracellular biological and mechanical signals ${ }^{2}$. Previous studies have identified two main types of primary cilia based on the positional characteristics of ciliary axonemes: surface cilia and subsurface cilia ${ }^{3,4}$. Most polarized epithelia often display cilia whose axoneme projects outward from the cell surface. Subsurface cilia, which are enclosed in deep, narrow pits created by membrane invagination, are commonly found in fibroblasts, smooth muscle cells and retinal pigment epithelia. Interestingly, spatial confinement or centriole subdistal appendages can transform subsurface cilia into surface cilia ${ }^{5,6}$. Primary cilia have also been reported to change their length in response to changes in serum concentration in the medium, which indicates that they may help regulate the balance between cell growth and quiescence ${ }^{7}$. As such, it is clear that a better morphological analysis of primary cilia in living cells is required to understand their functional complexity.

Scanning ion conductance microscopy (SICM) uses a glass nanopipette to acquire nanoscale live-cell topographical images in a solution ${ }^{8,9}$. Hopping-mode SICM and related techniques allow non-contact imaging because the nanopipette is withdrawn to a position well above the sample before moving to the next position laterally ${ }^{10-12}$. Recently, a low-cost, large field-of-view SICM has been developed for studying nanoparticle-cell membrane interactions ${ }^{13}$. Combining SICM with fluorescence microscopy allows targeting of specific cellular processes at the membrane interface ${ }^{14-16}$. Unlike atomic force microscopy, SICM is not limited by the cell's elastic modulus and can maintain the image quality for a longer period of time ${ }^{17}$. In addition, SICM images are comparable to those obtained using scanning electron microscopy (SEM), even when there are steep slopes on the cell surface ${ }^{18}$. By monitoring the current throughout the entire process, the topography and surface charge of cultured cells can be simultaneously probed ${ }^{19}$. Furthermore, SICM has been customized for functional imaging by employing double-barrel nanopipettes ${ }^{20,21}$. However, the SICM imaging of primary cilia is challenging because the cilia cell surfaces usually have large aspect ratios and shapes similar to microvilli that can be found over nearly the entire the cell surface. In this research, we have characterized the structure of primary cilia induced by serum starvation from two different cell types by combining SICM with fluorescence microscopy.

\section{Experimental Section}

Cell culture. Human retinal pigment epithelium-1 (RPE-1) cells were a kind gift from Dr. Ching-Hwa Sung (Weill Cornell Medical College, USA) ${ }^{22}$. Madin-Darby canine kidney (MDCK) cells were obtained from the Riken Cell Bank (Ibaraki, Japan). NIH3T3 cells were obtained from the Riken Cell Bank (Ibaraki, Japan). RPE-1 cells that stably express green fluorescent protein (GFP)-tagged mouse Smoothened (Smo) (Smo- 
GFP RPE-1) were established according to a previously reported method ${ }^{23}$. In brief, RPE-1 cells were transfected with pEGFP-mSmo plasmid DNA (Addgene) by Neon transfection system (Thermo Fisher Scientific), and Smo-GFP-positive cells were selected in the presence of G418 $400 \mu \mathrm{g} / \mathrm{mL}$. RPE-1 cells were maintained in DMEM-F12 (Wako) supplemented with 10\% fetal bovine serum (FBS) and 1 mM sodium pyruvate. MDCK cells were maintained in DMEM supplemented with $10 \%$ FBS. To induce cilium formation, cells were grown to confluence in basic cell culture conditions and then starved of serum for an additional $48 \mathrm{~h}$. NIH3T3 cells were maintained in DMEM supplemented with $10 \%$ FBS. To induce cilium formation, cells were grown to confluence in basic cell culture conditions and then starved of serum for an additional $48 \mathrm{~h}$.

Confocal fluorescence microscopy imaging. The RPE-1 and MDCK cells were plated on glass coverslips. Prior to imaging, cells were washed with PBSc/m (phosphate buffered saline supplemented with $0.2 \mathrm{mM}$ $\mathrm{Ca}^{2+}$ and $2 \mathrm{mM} \mathrm{Mg}^{2+}$ ) and fixed with $4 \%$ paraformaldehyde for $10 \mathrm{~min}$ at room temperature. The fixed cells were blocked in PBS containing 5\% BSA and $0.25 \%$ Triton X-100 for 30 min. The cells were further incubated with a mouse monoclonal antibody against acetylated $\alpha$-tubulin (Ac-Tub, Sigma) for $1 \mathrm{~h}$ and with Alexa-488-conjugated goat anti-mouse IgG (Thermo Fisher Scientific) for 45 min. Vybrant Dil (Thermo Fisher Scientific) or ActinRed 555 (Thermo Fisher Scientific) was co-treated with the second antibody. The nucleus was stained with 4,6-diamidino-2-phenylindole (DAPI). The fluorescent signals were acquired using a confocal laser-scanning microscope (LSM-780) equipped with a 63x/N.A. 1.4 oil objective lens (Carl Zeiss, Oberkochen, Germany) that enabled the acquisition of images every $0.5 \mu \mathrm{m}$ along the Zaxis. The images were processed using the "Maximum intensity projection" setting of the ZEN2011 software (Carl Zeiss).

SICM imaging. To simultaneously obtain the fluorescence and topographic images of primary cilia, the SICM system was mounted on a Nikon inverted fluorescence microscope (ECLIPSE Ti-S). SICM principles and instrumentation have been described in previous report ${ }^{24}$. SICM nanopipettes were fabricated by pulling borosilicate glass capillaries (inner diameter $=0.58 \mathrm{~mm}$, outer diameter $=1.00 \mathrm{~mm}$ ) using a $\mathrm{CO}_{2}$ laser puller. The internal radius of the nanopipette was approximately $50 \mathrm{~nm}$. The nanopipettes were backfilled with the same solution as that used for the bath. Two $\mathrm{Ag} / \mathrm{AgCl}$ electrodes were placed into the nanopipette and the bath, respectively. The ion current flowing through the nanopipettes was measured with an applied bias potential of $200 \mathrm{mV}$ between them. The theoretical approach curve was calculated according to the method used in a previous report ${ }^{25}$. 


\section{Results and discussion}

We first observed the structure of primary cilia from cultured RPE-1 and MDCK cells using conventional confocal microscopy. At 2 days post-confluence, more than $80 \%$ of RPE-1 cells exhibited a single primary cilium that was detected by immunofluorescence using an antibody against Ac-Tub in the presence of a cell labeling dye: Vybrant Dil (Fig. 1A). Some cilia appeared to be localized within the cell membrane (indicated by arrows in Fig. 1A), which would be consistent with the cilium being embedded within a membrane (ciliary) pocket. In contrast, the majority of primary cilia in MDCK cells stained with F-actin probe (ActinRed 555) were clearly protruding into the extracellular space (indicated by arrows in Fig. 1B). However, it should be noted that due to limited z-axis resolution of confocal microscopy, the presence of cilia either partially or entirely embedded in membrane pockets could not be excluded nor confirmed.

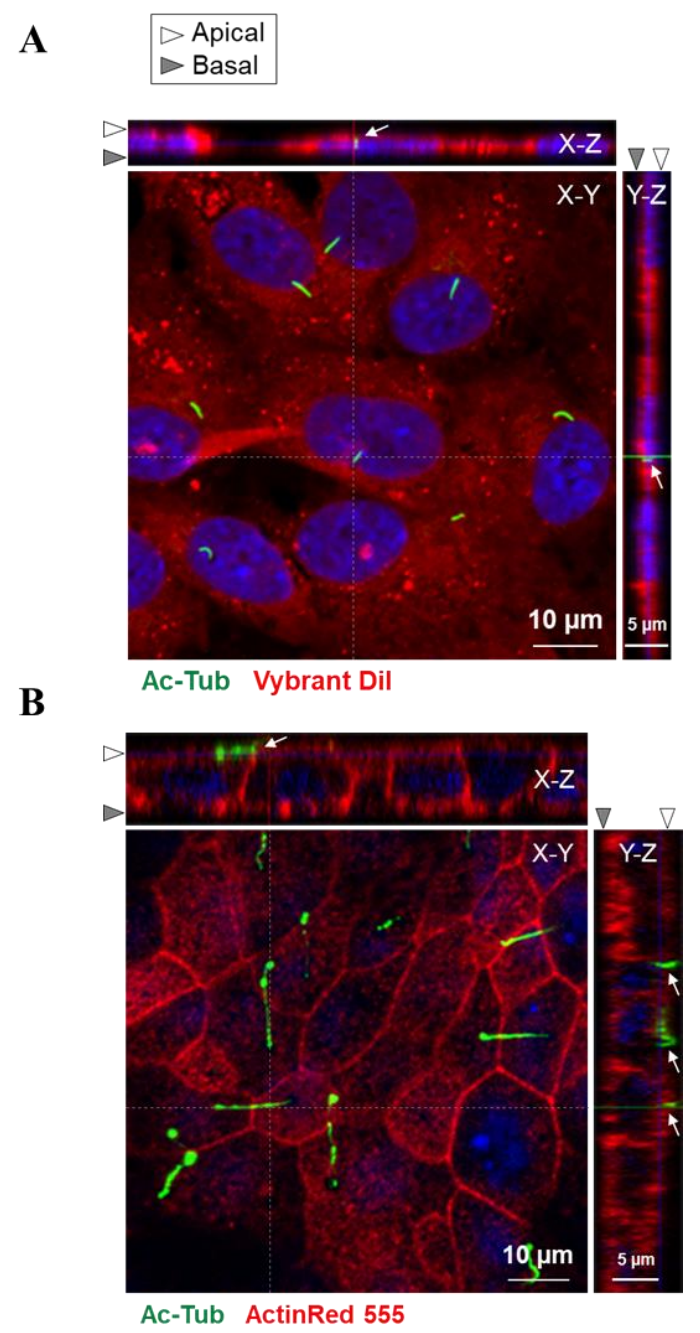

Figure 1. Confocal fluorescence images of Ac-Tub labeled primary cilia (green) from human retinal pigment epithelium-1 (RPE-1) cells (A) and Madin-Darby canine kidney (MDCK) cells (B). The RPE-1 and 
MDCK cells were co-labeled with Vybrant Dil (A; red) and ActinRed 555 (B; red), respectively. XZ and YZ optical sections illustrate cilia positioned below the cell surface in RPE-1 cells and above the cell surface in MDCK cells (arrows), respectively. The white and gray arrows indicate the apical and basal sides, respectively.

The topography of primary cilia was subsequently examined using SICM combined with fluorescence microscopy. To identify the position of the cilia, we first used the Smo-GFP RPE-1 cell line, which stably expresses the GFP-tagged cilia-specific protein-coupled receptor Smoothened ${ }^{23}$. Typical fluorescence and SICM images are presented in Figs. $2 \mathrm{~A}$ and 2B, respectively. The SICM image shows no structure matching the fluorescently marked cilium, suggesting this particular cilium was not exposed to the extracellular space. The cilia of the Ac-Tub labeled MDCK cells were then examined. Fig. $2 \mathrm{C}$ shows a representative fluorescence image of a primary cilium from a MDCK cell. A SICM image of the MDCK cell surface indicated that the cilium was approximately $7 \mu \mathrm{m}$ in length and $0.5 \mu \mathrm{m}$ in diameter (Fig. 2D).

A

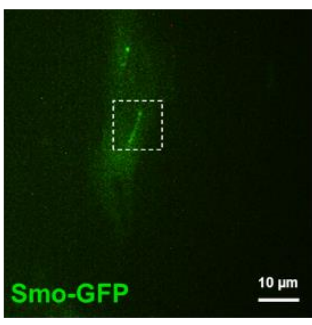

C

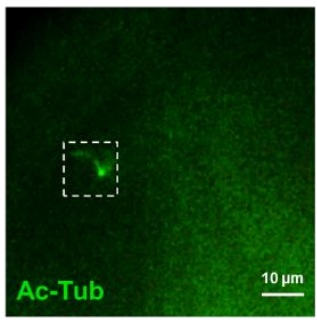

B

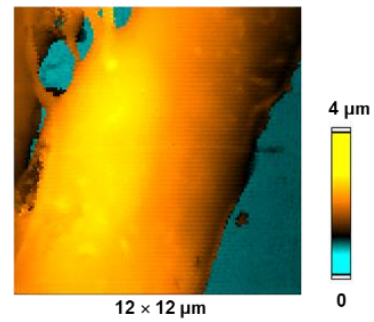

D

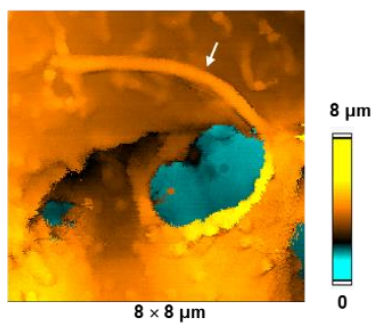

Figure 2. A comparison of a subsurface and a surface cilium. (A) Fluorescence image of primary cilium from a green fluorescent protein (GFP)-tagged Smoothened (Smo) (Smo-GFP) RPE-1 cell. (B) Scanning ion conductance microscopy (SICM) topographical image of the dashed region in (A). Consistent with the feature of subsurface cilia, the cell surface was smooth. (C) Fluorescence image of the Ac-Tub labeled primary cilium from an MDCK cell. (D) SICM topographical image of the dashed region in (C). The white arrow indicates the primary cilium. 
$\mathbf{A}$
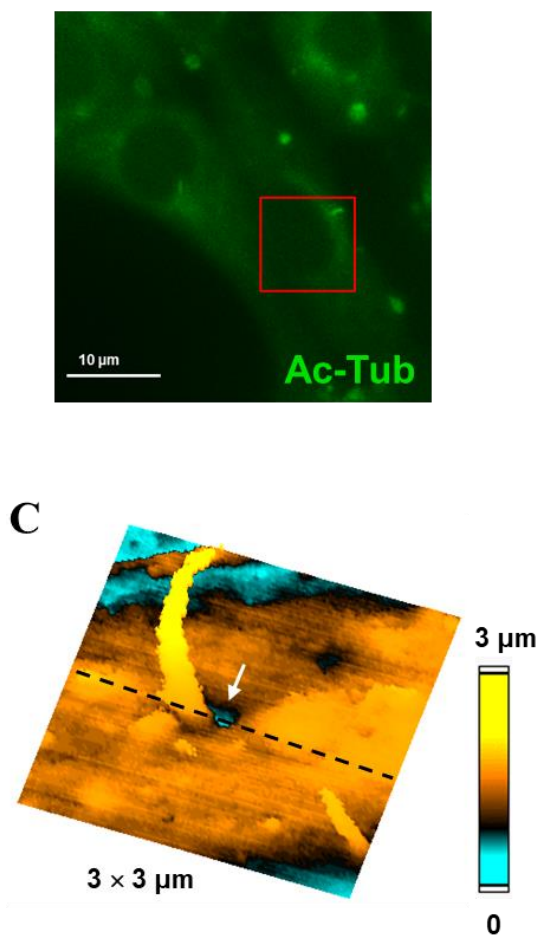

B

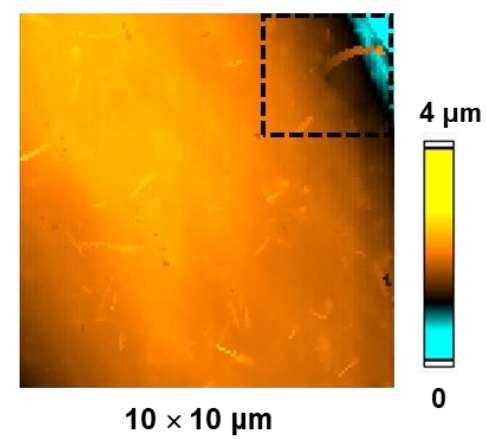

D

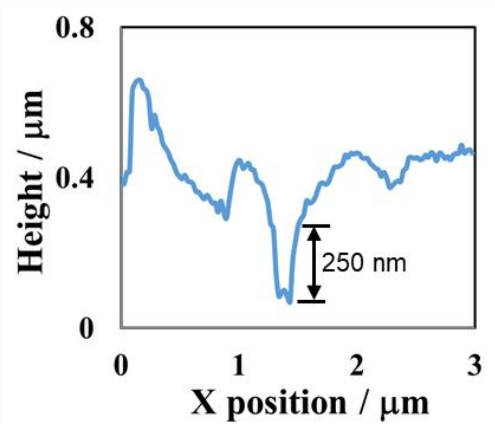

Figure 3. Visualization of the ciliary pocket from a NIH3T3 cell. (A) Fluorescence image. (B) SICM topographical image of the red region in (A). (C) High resolution SICM topographical image of the black dashed region in (B). The white arrow indicates the ciliary pocket. (D) Cross section at the same scan line marked in (C).

The ciliary pocket is an invagination of the plasma membrane which surrounds the cilia axoneme. Tracking this specific membrane domain will help to gain a better understanding of ciliary resorption ${ }^{26}$. However, this nanoscale structure cannot be identified by confocal microscopy. We have used the primary cilia of NIH3T3 cells as a model to investigate the structure of the ciliary pocket (Fig. 3A). The SICM image shows that $3 \mathrm{~T} 3$ cells also grow surface cilia (Fig. 3B). The high resolution SICM successfully detected the ciliary pocket at the base of the cilium (Fig. 3C). Cross section of the topographic image shows the depth of the ciliary pocket is about $250 \mathrm{~nm}$ (Fig. 3D). 
A

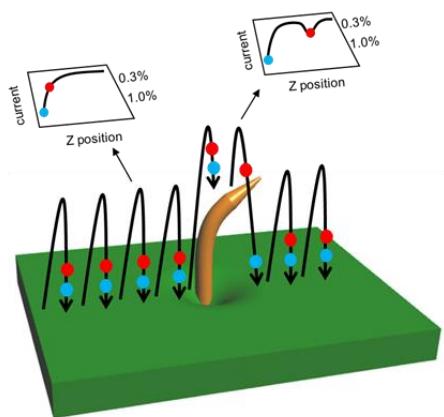

C

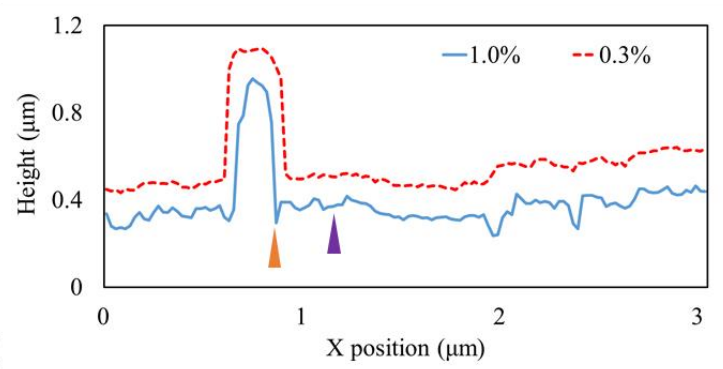

$\mathbf{E}$

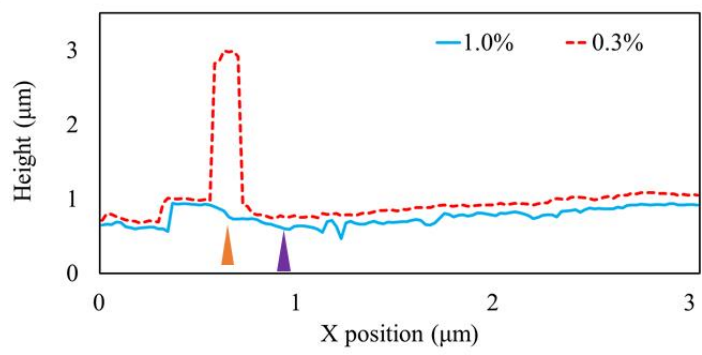

B
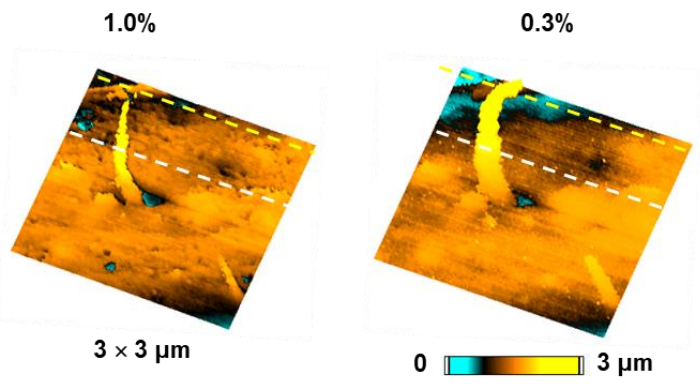

D

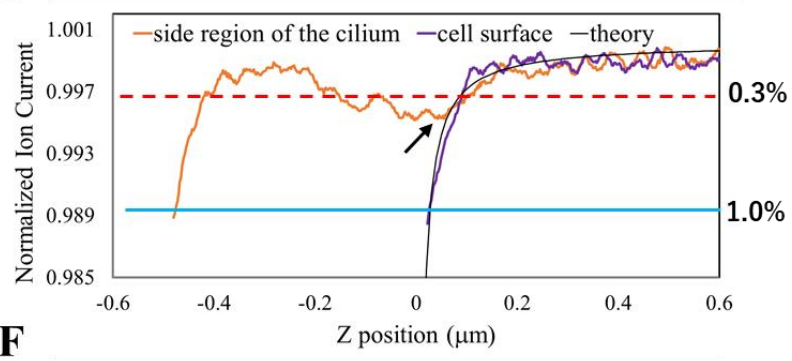

$\mathbf{F}$

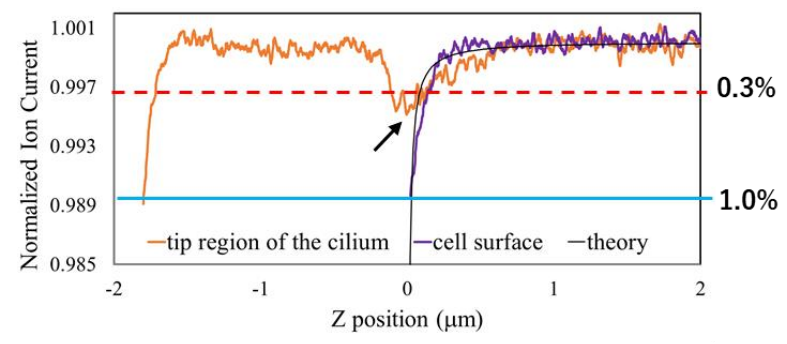

Figure 4. Effect of set point when scanning the primary cilium. (A) The principle of topography reconstruction. (B)SICM image of $1.0 \%$ set point (left) and reconstructed image of $0.3 \%$ set point (right). The dashed lines indicate the scan lines used to cross section analysis. (C) Cross sections at the white dashed lines marked in (B). The orange and purple arrows indicate the points of the side region of the cilium and cell surface for current-distance profile analyses. (D) Current-distance profiles at points marked in (C). " 0 " in Z position axes are determined by a theoretical approach curve of a $50 \mathrm{~nm}$ nanopipette. The black arrowhead indicates the point of pipette-cilium collision. (E) Cross sections at the yellow dashed lines marked in (B). The orange and purple arrows indicate the points of the tip region of the cilium and cell surface for current-distance profile analyses. (F) Typical current-distance profiles at points marked in (E). The black arrow indicates the point of pipette-cilium collision.

Although the hopping mode SICM is an ideal tool for non-contact imaging, several factors have been reported to affect image quality ${ }^{27,28}$. The relation between set point and topography has been examined by simulation and model biological samples ${ }^{29}$. A series of scans of the identical region are required to ap- 
preciate the details of the relationship. This is however extremely difficult to achieve because piezo drift always occurs during the scanning process. As a feature of hopping-mode SICM, spatial current-distance profiles can be recorded at each scan point ${ }^{30}$. To address the effect of set point on primary cilia imaging, we reconstructed topographic images of different set points based on current-distance profiles (Fig. 4A). We scanned the surface cilium from NIH3T3 cells at $1.0 \%$ set point and reconstructed topographic images (Movie 1). Figure 4B shows the SICM image of $1.0 \%$ set point and the reconstructed image of $0.3 \%$ set point. The reconstructed image is comparable to the one obtained by the actual scan at the $0.3 \%$ set point (Fig. 3C). Overall, obvious differences depending on the set point were observed. As the set point is increased, the diameter and length of the cilium decreased. This indicates the value of set point has a significant effect on the side and tip region of the cilium. To explain the effect in detail, we analyzed cross sections of topographic images and current-distance profiles at different parts of the cilium. Compared with the reconstructed cross section of the $0.3 \%$ set point, the $1.0 \%$ set point made the nanopipette position closer to the sample surface and caused artifacts at the side region of the cilium (Fig. 4C). Figure 4D shows the typical current-distance profiles at the side region of the cilium and the cell surface. At the cell surface, the ion current uniformly decreased during the nanopipette approach until the signal reached the set point. In contrast, at the side region of the cilium, the ion current decreased $0.3-0.4 \%$ but recovered during further approach and then decreased again until the signal reached the set point at the cell surface. Such a double-dip current-distance profile may be caused by pipette-cilium collision. Similar double-dip responses were also observed at the tip region of the cilium (Fig. 4F). As a result, the $1.0 \%$ set point failed to detect the tip region of the cilium but could be reconstructed at the $0.3 \%$ set point (Fig. 4E).

By imaging the primary cilia from RPE-1 and MDCK cells, we have demonstrated that SICM can be used as a simple tool to distinguish nanoscale protrusions with large aspect ratios on the cell surface. In addition, these studies have emphasised the importance of an appropriate set point for visualizing a surface primary cilium.

\section{ASSOCIATED CONTENT}

Supporting Information

Changes in primary cilium geometry according to different set points (MP4)

\section{AUTHOR INFORMATION}




\section{Corresponding Author}

* Tel: +81 76234 4866; e-mail: yasufumi@se.kanazawa-u.ac.jp

\section{ACKNOWLEDGMENT}

Development of Systems and Technology for Advanced Measure-ment and Analysis from AMED (The Japan Agency for Medical Research and Development)-SENTAN, PREST from the Japan Science and Technology Agency (JST), Grant-in-Aid for Young Scientists (A) (15H05422), Grant-in-Aid for Young Scientists (B) (15K20856) from the Japan Society for the Promotion of Science (JSPS), Takeda Science Foundation (to M.S.), Nakatani Foun-dation (to Y.T) and Kanazawa University CHOZEN Project are all thankfully acknowledged for financial sup-port.

\section{REFERENCES}

(1) Davenport, J. R.; Yoder, B. K. Am. J. Physiol. Ren. Physiol. 2005, 289, F1159-F1169.

(2) Christensen, S. T.; Pedersen, L. B.; Schneider, L.; Satir, P. Traffic 2007, 8, 97-109.

(3) Farnum, C. E.; Wilsman, N. J. Dev. Dyn. 2011, 240, 2405-2431.

(4) Molla-Herman, A.; Ghossoub, R.; Blisnick, T.; Meunier, A.; Serres, C.; Silbermann, F.; Emmerson, C.; Romeo, K.; Bourdoncle, P.; Schmitt, A.; Saunier, S.; Spassky, N.; Bastin, P.; Benmerah, A. J. Cell Sci. 2010, 123, 1785-1795.

(5) Pitaval, A.; Tseng, Q.; Bornens, M.; Théry, M. J. Cell Biol. 2010, 191, 303-312.

(6) Mazo, G.; Soplop, N.; Wang, W. J.; Uryu, K.; Tsou, M. F. B. Dev. Cell 2016, 39, 424-437.

(7) Yeh, C.; Li, A.; Chuang, J. Z.; Saito, Ca'ceres, A.; Sung, C. H. Dev. Cell 2013, 26, 358-368.

(8) Takahashi, Y.; Kumatani, A.; Shiku, H.; Matsue, T. Anal. Chem. 2017, 89, 342-357.

(9) Shevchuk, A. I.; Gorelik, J.; Harding, S. E.; Lab, M. J.; Klenerman, D.; Korchev, Y. E. Biophys. J. 2001, 81, $1759-1764$.

(10) Mann, S. A.; Hoffmann, G.; Hengstenberg, A.; Schuhmann, W.; Dietzel, I. D. J. Neurosci. Methods 2002, 116, $113-117$.

(11) Novak, P.; Li, C.; Shevchuk, A. I.; Stepanyan, R.; Caldwell, M.; Hughes, S.; Smart, T. G.; Gorelik, J.; Ostanin, V. P.; Lab, M. J.; Moss, G. W. J.; Frolenkov, G. I.; Klenerman, D.; Korchev, Y. E. Nat. Methods 2009, 6, 279-281.

(12) Ida, H.; Takahashi, Y.; Kumatani, A.; Shiku, H.; Matsue, T. Anal. Chem. 2017, 89, 6015-6020.

(13) Gesper, A.; Hagemann, P.; Happel, P. Nanoscale 2017, 9, 14172-14183.

(14) Shevchuk, A. I.; Hobson, P.; Lab, M. J.; Klenerman, D.; Krauzewicz, N.; Korchev, Y. E. Pflugers Arch. Eur. J. Physiol. 2008, 456, 227-235.

(15) Nikolaev, V. O.; Moshkov, A.; Lyon, A. R.; Miragoli, M.; Novak, P.; Paur, H.; Lohse, M. J.; Korchev, Y. E.; Harding, S. E.; Gorelik, J. Science. 2010, 327, 1653-1657.

(16) Nashimoto, Y.; Takahashi, Y.; Ida, H.; Matsumae, Y.; Ino, K.; Shiku, H.; Matsue, T. Anal. Chem. 2015, 87, $2542-2545$.

(17) Seifert, J.; Rheinlaender, J.; Novak, P.; Korchev, Y. E.; Schäffer, T. E. Langmuir 2015, 31, 6807-6813.

(18) Ushiki, T.; Nakajima, M.; Choi, M.; Cho, S.-J.; Iwata, F. Micron 2012, 43, 1390-1398.

(19) Page, A.; Perry, D.; Young, P.; Mitchell, D. A.; Frenguelli, B. G.; Unwin, P. R. Anal. Chem. 2016, 88, 10854-10859.

(20) Takahashi, Y.; Shevchuk, A. I.; Novak, P.; Zhang, Y.; Ebejer, N.; MacPherson, J. V.; Unwin, P. R.; Pollard, A. J.; Roy, D.; Clifford, C. A.; Shiku, H.; Matsue, T.; Klenerman, D.; Korchev, Y. E. Angew. Chemie - Int. Ed. 2011, 50, 9638-9642.

(21) Zhou, L.; Gong, Y.; Sunq, A.; Hou, J.; Baker, L. A. Anal. Chem. 2016, 88, 9630-9637.

(22) Li, A.; Saito, M.; Chuang, J.-Z.; Tseng, Y.-Y.; Dedesma, C.; Tomizawa, K.; Kaitsuka, T.; Sung, C.-H. Nat. Cell Biol. $2011,13,402-411$.

(23) Kim, J.; Lee, J. E.; Heynen-Genel, S.; Suyama, E.; Ono, K.; Lee, K.; Ideker, T.; Aza-Blanc, P.; Gleeson, J. G. Nature 2010, 464, 10481051. 
(24) Takahashi, Y.; Ito, K.; Wang, X.; Matsumae, Y.; Komaki, H.; Kumatani, A.; Ino, K.; Shiku, H.; Matsue, T. Electrochemistry 2014, 82, 331-334.

(25) Edwards, M. A.; Williams, C. G.; Whitworth, A. L.; Unwin, P. R. Anal. Chem. 2009, 81, 4482-4492.

(26) Saito, M.; Otsu, W.; Hsu, K.; Chuang, J.; Yanagisawa, T.; Shieh, V.; Kaitsuka, T.; Wei, F.; Tomizawa, K.; Sung, C. EMBO Rep. 2017, $18,1460-1472$.

(27) Thatenhorst, D.; Rheinlaender, J.; Schäffer, T. E.; Dietzel, I. D.; Happel, P. Anal. Chem. 2014, 86, 9838-9845.

(28) Rheinlaender, J.; Schäffer, T. E. Anal. Chem. 2015, 87, 7117-7124.

(29) Del Linz, S.; Willman, E.; Caldwell, M.; Klenerman, D.; Fernández, A.; Moss, G. Anal. Chem. 2014, 86, 2353-2360.

(30) Takahashi, Y.; Ida, H.; Matsumae, Y.; Komaki, H.; Zhou, Y.; Kumatani, A.; Kanzaki, M.; Shiku, H.; Matsue, T. Phys. Chem. Chem. Phys. 2017, 19, 26728-26733. 